Philosophie ANTIQUE

\section{Philosophie antique}

Problèmes, Renaissances, Usages

$9 \mid 2009$

Néoplatonisme

\title{
À propos du choix d'une variante chez Plotin
}

Ennéades, V, 3 [49], 7, 31

Pierre Thillet

\section{CpenEdition}

Journals

Édition électronique

URL : https://journals.openedition.org/philosant/2605

DOI : 10.4000/philosant.2605

ISSN : 2648-2789

Éditeur

Éditions Vrin

\section{Édition imprimée}

Date de publication : 13 novembre 2009

Pagination : 71-79

ISBN : 978-2-7574-0124-8

ISSN : $1634-4561$

\section{Référence électronique}

Pierre Thillet, « À propos du choix d'une variante chez Plotin », Philosophie antique [En ligne], 9| 2009, mis en ligne le 25 juillet 2019, consulté le 07 décembre 2022. URL : http://journals.openedition.org/ philosant/2605 ; DOI : https://doi.org/10.4000/philosant.2605

\section{(c) (i) (3)}

Creative Commons - Attribution - Pas d'Utilisation Commerciale - Pas de Modification 4.0 International - CC BY-NC-ND 4.0

https://creativecommons.org/licenses/by-nc-nd/4.0/ 


\author{
À PROPOS DU CHOIX D'UNE VARIANTE CHEZ PLOTIN \\ Ennéades, V, 3 [49], 7, 31 \\ Pierre THILLET \\ Université de Paris I Panthéon-Sorbonne
}

Les éditeurs de textes grecs n'ont pas toujours raison de respecter à la lettre la formule recentiores deteriores. Ce n'est pas le lieu de rassembler des exemples où, d'évidence, la leçon d'un recentior, ainsi tel manuscrit du XVe siècle, est préférable à celle qu'offrent des témoins plus anciens.

Un passage de Plotin permet d'illustrer cette remarque. Il ne s'agit pas, certes, d'une proposition nouvelle : le choix d'une leçon empruntée à un recentior a déjà été fait par un savant lecteur des Ennéades ${ }^{1}$. Il manque cependant, à ce choix, une justification ${ }^{2}$ que la présente note, je l'espère, cherche à donner.

Dans un passage d'apparence assez peu difficile d'Ennéade V, 3 [49], 7,31 , un participe fait problème. Les anciens éditeurs s'en sont accommodés, encore que le sens puisse paraitre peu satisfaisant. La phrase est la suivante :

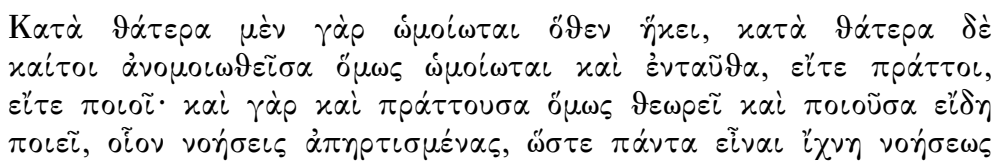

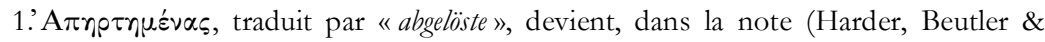
Theiler 1960, Vb, p. 378) «abgetrennt». Un tel choix parait fort audacieux pour qui admet comme principe la formule recentiores deteriores, puisque J est visiblement un codex du $\mathrm{XV}^{\mathrm{e}}$ siècle.

2. R. Harder donne cependant une note (Harder, Beutler \& Theiler 1960, Vb, p. 378).

Philosophie antique, $\mathrm{n}^{\circ} 9$ (2009), 71-79 


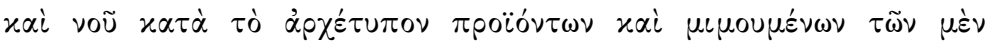

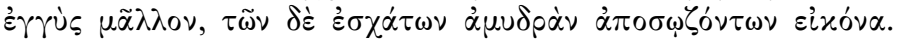

Bréhier traduit: «en produisant, elle (scil. l'intelligence) produit des formes, qui sont comme des pensées bien ajustées $»^{3}$. Cette traduction

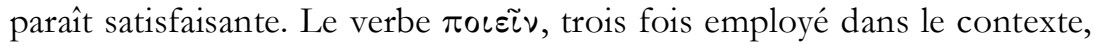
rend cohérente l'idée d'un achèvement parfait exprimée par le verbe

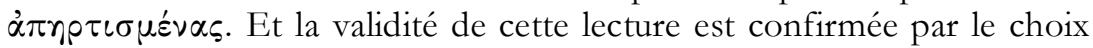
qu'en a fait A.H. Armstrong, qui traduit « and in its production it produces forms, which are like intellections carried out in practice $»^{4}$. Or Armstrong connaît le manuscrit recentior qu'ignorait Bréhier, et qui offre une autre leçon. Il est vrai que les éditeurs de Plotin, P. Henry et H.R. Schwyzer ont con-

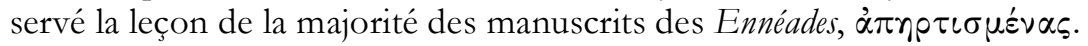

Si Plotin se plaît ainsi à souligner que les « pensées » ${ }^{5}$, peut-être mieux les « intellections» (pour conserver dans la traduction quelque chose du rapport de ces «pensées » avec le voũ $\varsigma^{6}$ ), sont bien agencées, ce pourrait être dans l'intention d'en souligner la perfection. Mais qui pourrait penser que ce qui vient du voũ s puisse présenter quelque imperfection?

Cependant le cod. J - dont les particularités, signalées par le P. Henry, suscitent parfois la suspicion - offre une leçon qu'il est le seul de sa

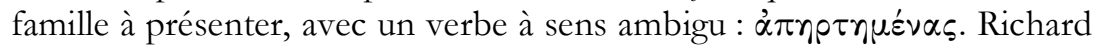
Harder a choisi cette leçon ${ }^{7}$. C'est aussi la leçon qu'adopte Bertrand Ham en lui donnant un sens qu'il justifie longuement dans son commentaire ${ }^{8}$. Selon ce dernier, les idées produites par l'âme sont « séparées » de l'intelligence. N'y a-t-il pas de quoi s'étonner ? Il semblerait que les pensées ${ }^{9}$ doivent rester, en quelque façon, liées à l'intelligence. C'est du moins ce qu'on attendrait d'une doctrine où tout émane du principe unique. Suffirait-il donc d'ajouter une négation devant le participe que l'on traduit par « séparées ${ }^{10}$ ? Mais il convient de ne pas se laisser aller à la première impression.

3. Bréhier 1931, ad loc.

4. Armstrong 1984, ad loc.

5. Ham 2000, «détachées», n. 81, p. 65, et commentaire, p. 161-162, traduit par «pensées détachées » et il justifie cette traduction p. 161-162.

6. Pour garder la traduction de Bréhier.

7. Auquel cas on traduirait par "non séparées », en conservant le sens donné au participe.

8. Voir supra note 5.

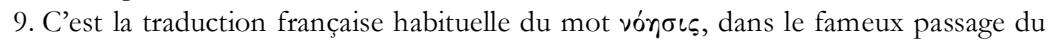
livre $\Lambda$ de la Métaphysique d'Aristote, cité infra note 12.

10. Auquel cas on traduirait par "non séparées ", en conservant le sens donné au participe. 
Ce passage des Ennéades a été lu, et non pas «traduit», en arabe par l'auteur de l'Épitre sur la science divine ${ }^{11}$. Malheureusement l'adaptation est en cet endroit si éloignée du texte grec qu'il n'est pas possible de savoir quels mots l'auteur de l'Épitre avait sous les yeux.

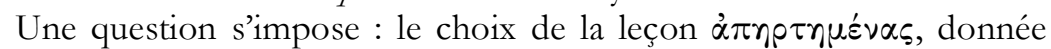
par le seul ms. J, est-il justifié ? Bréhier, qui ne connaissait pas le ms. J, a édité $\alpha \pi \eta \rho \tau \iota \sigma \mu \varepsilon ́ v \alpha \varsigma$ et traduit par «bien ajustées», s'agissant des

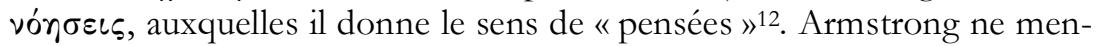

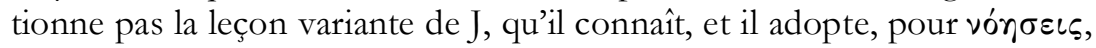
le sens de «intellections $»^{13}$. Mais si l'on fait choix de la leçon offerte par le ms. J, le sens à donner au verbe $\alpha \dot{\alpha} \alpha \rho \tau \tilde{\alpha} \sigma \vartheta \alpha \iota$ devient problématique. On peut comprendre en effet " être séparé », mais aussi «être suspendu » ou même «être rattaché » (auquel cas, faut-il penser, le verbe ne serait pas employé au sens absolu, mais demanderait une préposition).

On voit combien le choix, proposé par Theiler, de la leçon offerte par le seul manuscrit J est loin d'apparaittre comme une alternative satisfaisante à la leçon que présentent tous les autres manuscrits. L'apparat critique des éditions de Henry et Schwyzer, où figure la leçon variante du ms. J, marque bien le caractère solitaire de sa lecture en cet endroit : « 7,

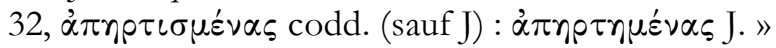

Unique témoin de cette leçon, le manuscrit J présente aussi le défaut d'être un recentior. Malgré la singularité de J, Parisinus gr. 2082, qui date du $\mathrm{XV}^{\mathrm{e}}$ siècle, et dont les variantes seraient souvent, selon Paul Henry, des conjectures du copiste ${ }^{14}$, on serait d'abord tenté de choisir sa leçon, comme l'a fait Theiler, mais en la faisant précéder de la négation oủx. Les

11. Écrit attribué à Farabi dans quelques manuscrits. Il provient d'une traductionadaptation arabe des Ennéades. On en trouve une édition dans Badawi 1955, p. 165-183.

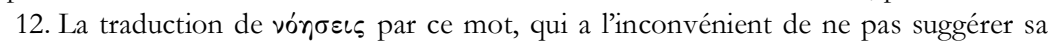
relation avec l'Intelligence (voũs), est justifiée par B. Ham (voir note 5), et elle est couramment utilisée. C'est, je crois, la traduction habituelle du passage de la Métaphysique

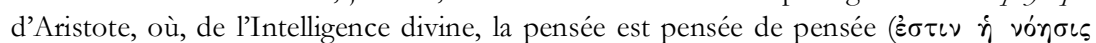

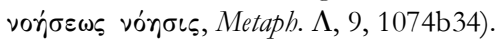

13. Voir supra note 4. Le texte grec reproduit, presque exactement, celui de l'editio major de Henry et Schwyzer 1977. La traduction «like intellections carried out in practice» ne semble pas plus satisfaisante, dans le contexte, que la traduction de Bréhier.

14. Voir Henry 1948, p. 135-148. Du ms. J (Parisinus gr. 2082), P. Henry disait qu'il « est sans doute le plus mal connu et le plus singulier» de tous les mss. de Plotin. (Henry 1948, p. 115), à quoi il ajoutait que «faire peu de cas de ces leçons aberrantes... c'est chercher à voir clair en se mettant un bandeau sur les yeux » (id. p. 116). J offre, certes, des «leçons solitaires», mais on ne saurait les condamner toutes comme purement conjecturales. P. Henry lui-même, en effet, signale justement, en III, 7, 9, 2, une leçon propre à J, qui se trouve confirmée par Simplicius (id. p. 146). 
« formes » produites par l'activité de l'âme intellective, sont peut-être des "pensées bien ajustées»(Bréhier), selon la leçon de la majorité des manuscrits, qui nous place dans une perspective, non pas sans doute cré-

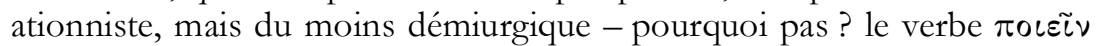
est utilisé deux fois dans la même ligne ${ }^{15}$ - mais il est bien plus probable qu'elles sont «non séparées ». Plotin, en conformité avec sa doctrine, ne peut qu'insister sur la continuité de l'intelligible avec ce qui est au dessous de lui, et qui provient de lui. Il récuse la « séparation » radicale entre le bas et le haut principiel ${ }^{16}$. Le chapitre 8 qui suit contribue à renforcer cette interprétation. L'âme "produit», mais ce qu'elle produit n'est en

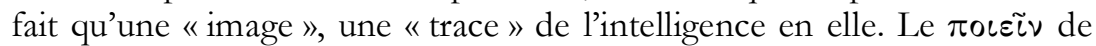
Plotin n'est pas «création ». On ne saurait se représenter le producteur comme l'artisan qui, une fois son travail mené à bien, se sépare de son œuvre dont l'existence est dès lors tout à fait indépendante. La présence du verbe $\alpha \pi \alpha \rho \tau i \zeta \varepsilon \iota \nu$ en VI, 3 [44], 4, 32, ne saurait contraindre à choisir ici la leçon des manuscrits autres que $\mathrm{J}^{17}$.

Dans cette perspective, il convient de s'interroger sur la présence du

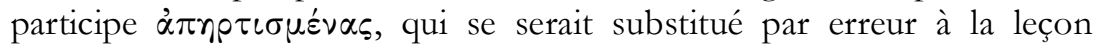

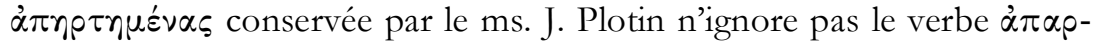

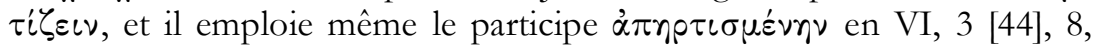
$23^{18}$. Il est notoire que ce même participe est très abondant chez des auteurs chrétiens, comme saint Jean Chrysostome, et dans les chaînes exégétiques. On pourrait voir ici le principe d'une faute : familier avec la graphie $\alpha \pi n \rho \tau \iota \sigma \mu \varepsilon^{\prime} v \alpha \varsigma$, un copiste ${ }^{19}$ aurait pu la substituer à ce qu'il aurait

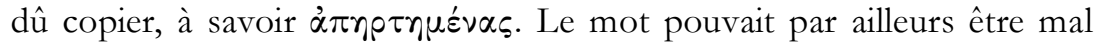
lisible sur le modèle.

Il convient de revenir sur cette difficulté. En effet, une conjecture permettrait de sauver une lecture controversée, en ajoutant une négation. On lirait <oủx> $\alpha \pi n \rho \tau \iota \sigma \mu \varepsilon ́ v \alpha \varsigma$, si l'on veut donner au verbe $\dot{\alpha} \pi \alpha \rho \tau i \zeta \varepsilon \iota \nu$ le sens de «séparer », qui semble avoir été son sens le plus fréquent. Mais

15. S'agirait-il de suivre Aristote contre Platon ? Le Stagirite, en effet, condamne la "séparation » $(\chi \omega \rho \iota \sigma \mu o ́ s)$ introduite par Platon entre les Idées et la réalité sensible. Mais le choix du verbe $\alpha \pi \alpha \rho \tau \tilde{\alpha} \nu$ par Plotin pourrait signifier son souci de ne pas suivre Aristote.

16. Porphyre, dans les Sentences, ignore $\alpha \pi \alpha \rho \tau i \zeta \varepsilon \iota \nu$ et emploie deux fois $\dot{\alpha} \pi \alpha \rho \tau \tilde{\alpha} \nu$ (40, $11 ; 43,39)$.

17. Il s'agit en ce passage de montrer l'exact ajustement de la forme à la matière.

18. Il est à noter qu'en ce lieu Plotin évoque la réalisation des substances par les qualités. L'implication noétique est donc fort lointaine.

19. Hypothèse aventurée. Les copistes, semble-t-il, étaient le plus souvent spécialisés, les uns copiant les Évangiles ou les textes des Pères de l'Église, les autres les textes venus des anciens païens. 
il convient de remarquer que ce verbe a aussi un autre sens, qui est même opposé, "suspendre ", "s'attacher à ». L'évidence saute aux yeux du lecteur de la Consolation à Apollonios, de Plutarque (Moralia, $105 \mathrm{E}$ ), où

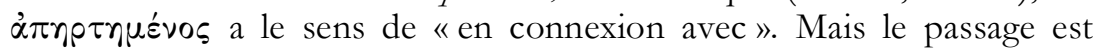
douteux et les derniers éditeurs, à la suite de Herten, corrigent le texte ${ }^{20}$.

Or il semble possible de savoir en quel sens Plotin emploie le verbe $\dot{\alpha} \pi \alpha \rho \tau \tilde{\alpha} \nu$. Et de façon plus heureuse, on constate que Plotin, en dehors de ce passage controversé, offre trois autres emplois du participe $\dot{\alpha} \pi \eta p-$

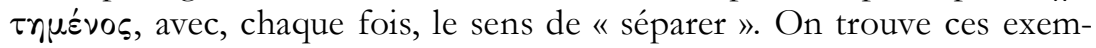
ples en IV, 3 [27], 12, 20 ; VI, 6 [34], 1, 17 ; VI, 8 [39], 18, 25. Il est à remarquer cependant que, chronologiquement, ces traités sont assez éloignés de notre traité [49].

En IV, 3 [27], 12, 20, la négation accompagne immédiatement le participe. Plotin évoque le statut des âmes individuelles, qui ne sont pas détachées du tout. Bréhier traduit: "elles n'agissent pas isolément»; Beutler: "nicht von ibm abgetrennt sind»; Armstrong: "they are not cut off from ». L'accord des traducteurs permet déjà de fixer le sens que le verbe a pour Plotin.

Dans le Traité sur les nombres, VI, 6 [34], 1, 17, où le participe àrnp-

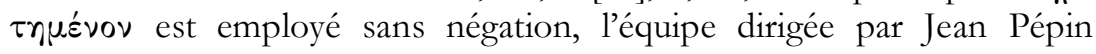
traduit : « si l'objet est divisé ». Bréhier avait déjà traduit par «se divise »; Armstrong : «by separation »; Beutler : « auseinandergerissen ».

Enfin, en VI, 8 [39], 18, 25, Sur la liberté et la volonté de l'Un, dans une comparaison célèbre, où l'un est comme le centre, et ce qui en vient comme les rayons d'un cercle, les rayons ne se détachent nullement du

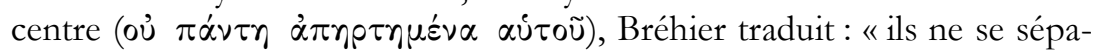
rent pas de lui »; Armstrong: "not at all cut off from it»; Beutler : "nicht von ibm getrennt sind».

Il reste toutefois que le sens du verbe est ambigu. Si nous examinons les emplois du participe avant Plotin (Platon n'en donne aucun exemple)

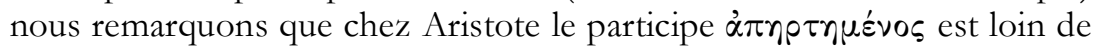
signifier toujours la «séparation ». Il a même, assez souvent, le sens de

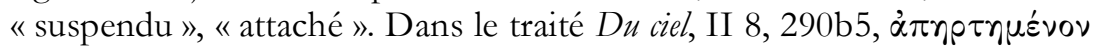
est traduit par Paul Moraux «appendices», et par Tricot «membre attaché ». Si, en Parties des animaux, I, 11, 657a13, Pierre Louis traduit par "détaché », et en IV, 13, 695b17 par "distincts », en revanche, en IV, 2, $676 \mathrm{~b} 17$, il traduit par «en rapport avec » (construit, il est vrai, avec ह̇ $\pi i$ ), et en IV, 12, 693a26, «attaché ».

20. Voir la Consolation à Apollonios, 105 E, \8, dans Defradas, Hani et Klaerr 1985, p. 50 . 
Ce sens de «attaché » paraît bien confirmé dans un texte plus tardif. Eusèbe de Césarée, en effet, l'emploie dans sa Préparation évangélique, en un lieu où le P. des Places a traduit par «dépendant de »21.

On en pourrait donc inférer que le verbe $\alpha \dot{\alpha} \alpha \rho \tau \tilde{\alpha} \nu$ n'a pas vraiment le sens de "séparer", mais qu'il signifie que le sujet auquel il s'applique se détache, et reste suspendu, rattaché à ce dont il s'écarte. À cet égard sont signifiants les emplois du participe dans certains traités biologiques d'Aristote. Le Stagirite en fait usage chaque fois qu'il s'agit de noter qu'un organe «se sépare » du corps, ou d'un autre organe, auquel il reste « rattaché ». Un pédoncule rattache ainsi les testicules au bas-ventre ${ }^{22}$, ou la vésicule biliaire au foie ${ }^{23}$. Dans d'autres descriptions anatomiques, on retrouve le même participe. Ainsi à propos du rapport entre cœur et poumon chez les serpents ${ }^{24}$. Dans sa description de la relation entre la denture et l'âge du cheval, un emploi du participe par Aristote mérite d'être signalé. La canine du cheval qu'on ne monte pas est grande, mais «déchaussée » ${ }^{25}$. Les oreilles des quadrupèdes sont «détachées 》 et audessus des yeux ${ }^{26}$. En revanche, les ailes des oiseaux sont «attachées aux bras $»^{27}$. Ce qui caractérise les poissons, c'est qu'il n'ont pas de membres «distincts $»^{28}$.

Cependant, chez Aristote encore, le participe apparait précisément pour signifier l'idée d'un éloignement qui implique une coupure. C'est le cas dans les Topiques, où un adverbe précise la signification ${ }^{29}$.

Cette revue tendrait à montrer la diversité des sens du verbe, sens qui semblent parfois contraires ${ }^{30}$. En fait, il convient de remarquer que, dans

21. Eusèbe de Césarée, Praep. ev. VI, 6, 22, 6 (Des Places 1980, p. 139).

22. Aristote, GA I, 2, 716b29-30 ; I, 13, 720a24 ; HA III, 1, 509b14-15, «pendants » traduisent Louis 1964, p. 70 et Tricot 1957, p. 158.

23. Aristote, $H A$ II, 45, 506b19.

24. Id. II, 17, 508a33. Décrivant l'anatomie du serpent, Aristote dit de son poumon qu'il est «largement détaché du cœur » (Louis 1964, p. 66), " complètement détaché » (Tricot 1957, vol. 1, p. 151).

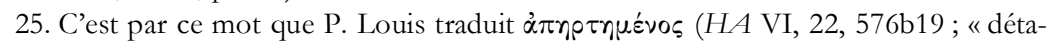
chée » Tricot 1957, vol. 2, p. 437). Il faut comprendre que la partie de la dent couverte d'émail est comme suspendue par la racine, alors visible, comme une sorte de tige. Le cheval que l'on monte a les dents usées par le mors, elles ne présentent pas cette particularité.

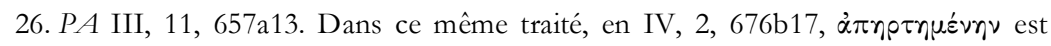
traduit par P. Louis 1956 « en rapport avec », façon d'éluder la traduction du participe.

27. Id. IV , 12, 693a26 ; le participe est construit avec $\dot{\alpha} \pi \dot{o}$, et certains mss. ont $\dot{\alpha} v \tau \dot{\text {. }}$

28. Id. IV 13, 695b17.

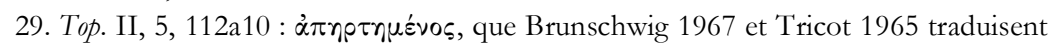
respectivement par « éloigné » et par « en dehors de ».

30. Un exemple notable dans la Préparation évangélique d'Eusèbe de Césarée, VI, 6, 22. 
tous les cas, l'idée de séparation n'est que relative ; elle n'exclut aucunement la possibilité d'un lien. La notion de distance signifiée par le préfixe n'efface pas l'idée de rattachement notée par la racine verbale.

Pour limité qu'il soit, l'inventaire auquel il a été procédé permet de donner tout son sens à la leçon du ms. J des Ennéades. Il devient tout à fait possible, dans notre passage de Plotin, de lire, avec le ms. J, malgré sa

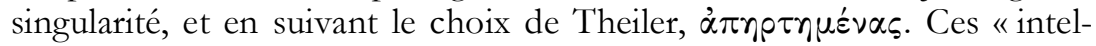
lections », qui sont " produites ", ne sont pas sans rapport avec les archétypes de l'intelligence, elles leur restent attachées, et n'en sont pas totalement indépendantes. La continuité entre l'intelligence et l'âme intellective paraît bien nettement maintenue.

Mais comme le contexte, dans le traité des Ennéades, n'est en rien aristotélicien, comme les trois emplois avérés du participe permettent de connaitre en quel sens il semble avoir été compris par Plotin, rien ne nous autorise à penser qu'on puisse lui donner ici, en V, 3 [49], un sens différent. Faut-il alors supposer que le participe ait été accompagné d'une négation très tôt omise par un copiste, lors de la transmission du texte ? On ferait alors l'hypothèse que c'est l'absence de négation qui aurait pu être l'occasion, pour un lecteur, de remplacer $\alpha \dot{\pi} \eta \rho \tau \eta \mu \varepsilon^{\prime} \alpha \varsigma$ par $\alpha \dot{\pi} \eta \rho-$ $\tau \iota \sigma \mu e^{\prime} v a \varsigma$. Mais ce sont là des hypothèses assez vaines.

Ce qui pourrait confirmer ainsi l'emploi d'un terme emprunté, c'est que Plotin le fait précéder de oĩov; on peut interpréter cela comme le signe que le mot doit être pris dans un sens qui n'est plus celui du concret auquel il est emprunté.

On peut voir une autre confirmation du sens à donner à ce passage, en y admettant la leçon du ms. J, en VI, 7 [38], 5, 29. L'âme de l'homme qui perçoit n'est pas en dehors de l'intelligible, car elle lui reste « attachée, comme suspendue à lui » ${ }^{31}$.

Il est notable que Plotin, à la différence de Platon, et peut-être comme en héritage de l'aristotélisme, n'admet pas ce qu'Aristote a explicitement condamné chez son maitre (selon l'interprétation qu'il en fait), à savoir la "séparation » ( $\chi \omega \rho \iota \sigma \mu o ́ s)$ des Idées ${ }^{32}$. Pour Plotin, les Idées ne sont pas même séparées totalement de la matière ${ }^{33}$, et cela sans qu'on

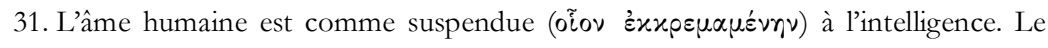
texte souligne l'attachement à l'intelligible.

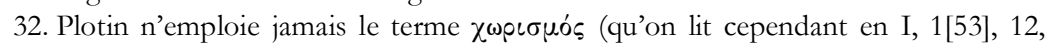
18 ; II, 9 [33], 6, 40 ; III, 6 [26], 5, 21 ; IV, 7 [2], 8, 20) pour signifier cette séparation des Idées. Il se borne à affirmer que l'intelligence n'est jamais totalement absente du sensible, voire de la matière. C'est bien là une exigence de la doctrine de l'émanation.

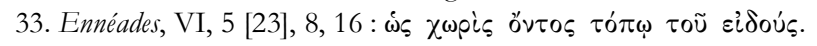


puisse évoquer un intermédiaire ${ }^{34}$. C'est l'intelligence qui reste en haut $\left(\alpha^{\prime} \nu \omega\right)$ en agissant en bas ( $\left.\varepsilon i \varsigma \tau \dot{\alpha} \tau \tilde{\eta} \delta \varepsilon\right)$, mais cette fois par l'intermédiaire de l'âme ${ }^{35}$. Il ne faut pas voir là une contradiction. L'intelligence conserve son statut primordial, et ne saurait «descendre». Ce sont les Idées qui confèrent au multiple la variété des formes.

Ces remarques contribuent à justifier le choix fait par Theiler de la leçon du Parisinus gr. 2082, manuscrit du XVe siècle. Ce ne serait pas là une conjecture de copiste, mais, vraisemblablement, une leçon ancienne, peut-être originelle.

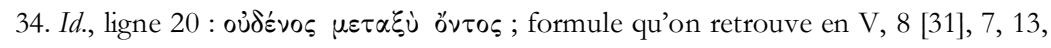

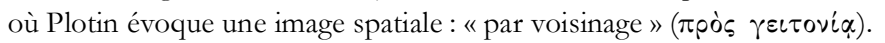

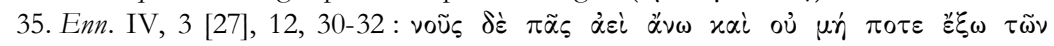

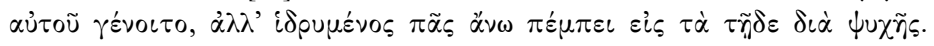




\section{BIBLIOGRAPHIE}

Armstrong, A.H. 1984 : Plotinus in seven volumes. 5, Enneads V. 1-9, with an English Translation by —, Cambridge, Mass., 1984 (The Loeb classical library, 444).

BADAwI, A. 1977 : Aflutin 'inda 'l-'Arab = Plotinus apud Arabes. Theologia Aristotelis et fragmenta quae supersunt. Collegit, edidit et prolegomenis instruxit —, Le Caire, 1955 (Islamica, 20), Kuwait, ${ }^{3} 1977$.

BrÉHIER, É. 1931 : Plotin, Ennéades. 5, texte établi et traduit par —, Paris, 1931 (Collection des Universités de France).

Brunschwig, J. 1967 : Aristote, Topiques. 1, Livres I-IV, texte établi et traduit par Paris, 1967 (Collection des Universités de France).

Defradas, J., J. HANi \& R. KLAERr 1985 : Plutarque, CEuvres morales. 2, Consolation à Apollonios; Préceptes de santé; Préceptes de mariage; Le banquet des sept sages; De la superstition, texte établi et traduit par — , Paris, 1985 (Collection des Universités de France).

Des Places, É. 1980 : Eusèbe de Césarée, La Préparation évangélique. Livres V, 18-36-VI; introduction, texte grec, traduction et annotation par —, Paris, 1980 (Sources chrétiennes, 266).

Ham, B. 2000 : Plotin, Traité 49 : V, 3, introduction, traduction, commentaire et notes, Paris, 2000 (Les Écrits de Plotin).

Harder, R ., R. Beutler \& W. Theiler 1960 : Plotins Schriften. 5, Die Schriften 46-54 der chronologischen Reihenfolge : a, Text und Übersetzung ; b, Anmerkungen, übersetzt von R. Harder; Neuarbeitung mit griechischen Lesetext fortgeführt von R. Beutler und W. Theiler, Hambourg, 1960 (Philosophische Bibliothek, 215 a-b).

Henry, P. 1948 : Études plotiniennes. II, Les manuscrits des Ennéades, Paris-Bruxelles, ${ }^{2} 1948$ (Museum Lessianum. Section philosophique, 21).

Henry, P. \& H.-R. Schwyzer, Plotini Opera, t. 2 : Enneades $I V$ - $V$, ediderunt - Oxford, 1977, 1982 (Scriptorum classicorum bibliotheca oxoniensis).

Louis, P. 1956 : Aristote, Les Parties des animaux, texte établi et traduit par —, Paris, 1956 (Collection des Universités de France).

- 1964 : Aristote, Histoire des animaux, 1, Livres I-IV, texte établi et traduit par —, Paris, 1964 (Collection des Universités de France).

- 1968 : Aristote, Histoire des animaux, 2, Livres V-VII, texte établi et traduit par —, Paris, 1968 (Collection des Universités de France).

TricOT, J. 1957 : Aristote, Histoire des animaux, nouvelle traduction avec introduction, notes et index, 2 vol., Paris, 1957 (Bibliothèque des textes philosophiques).

- 1965 : Aristote, Organon. 5, Les Topiques, traduction nouvelle et notes, nouvelle édition, Paris, 1965 (Bibliothèque des textes philosophiques). 